\title{
ON THE CONSTRUCTION OF HERMITIAN FROM LAGRANGIAN DIFFERENCE APPROXIMATIONS*
}

\author{
By MAHMUT TANRIKULU AND WILLIAM PRAGER \\ (Department of the Aerospace and Mechanical Engineering Sciences \\ University of California, San Diego La Jolla, California)
}

\begin{abstract}
It is shown how simple finite difference approximations to the Laplace operator in two- or three-dimensions can be combined to construct Hermitian finite difference approximations to the two- or three-dimensional Poisson equation $\Delta u=f$.
\end{abstract}

1. Introduction. This note is concerned with finite difference approximations to Poisson's equation, $\Delta u=f$, where $\Delta$ denotes the Laplace operator and $f$ is a given function. These finite difference approximations are called Lagrangian or Hermitian according to whether they involve only one or several values of $f$. (Collatz [1], who first stressed the accuracy obtainable with Hermitian approximations, coined the term "Mehrstellenverfahren".)

Whereas Lagrangian approximations of various degrees of precision are readily established, the construction of Hermitian approximations is less direct. Indeed, the Taylor expansions of $u$ and $\Delta u=f$ at a given node of a square or cubic grid must be evaluated for the neighboring nodes, and the resulting expressions must be combined in such a manner that all terms below a certain degree in the mesh width $h$ cancel out. A method of achieving this "Taylor cancellation" (called "Taylor Abgleich" by Collatz [1]) has been described by Meister and Prager [2]. While this method is straightforward, it requires a certain amount of algebraic work, which increases rapidly with the desired degree of precision.

The present note is meant to draw attention to the way in which readily obtainable Lagrangian approximations to $\Delta u$ can be combined to yield approximations to $\Delta^{2} u$ and Hermitian approximations to the equation $\Delta u=f$.

2. Lagrangian approximations to $\Delta u$ and $\Delta^{2} u$. Figure 1 shows the labelling of the nodes of a two-dimensional square grid in the neighborhood of the typical node 0 . Note that there is only one node labelled 0 , but there are four nodes each labelled 1, 2, 3 . The following discussion is restricted to symmetric difference operators in which the values of the function $u$ at equally labelled nodes appear with the same weight. The sum of the values of $u$ at all nodes labelled $i$ will be denoted by $u_{i}$. Similarly, $\Delta u_{i}$ will denote the sum of the values of $\Delta u$ at all nodes labelled $i$, and so on.

With this notation, the most familiar finite difference approximations to $\Delta u_{0}$ may be written in the forms

$$
h^{2} \Delta u_{0}=-4 u_{0}+u_{1}+h^{4} Q u_{0}+O\left(h^{6}\right),
$$

and

$$
12 h^{2} \Delta u_{0}=-60 u_{0}+16 u_{1}-u_{2}+h^{6} R u_{0}+O\left(h^{8}\right),
$$

*Received March 28, 1966. This research was supported by the Advanced Research Projects Agency (Project DEFENDER) and was monitored by the U. S. Army Research Office-Durham under Contract DA-31-124-ARO-D-257. 


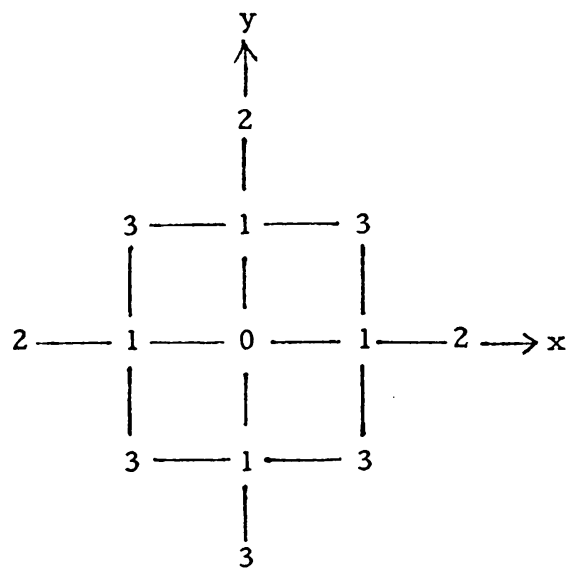

Fia. 1. Nodes of square grid and nodes in plane $z=0$ of cubic grid.

where

$$
Q=-\frac{1}{12}\left(\frac{\partial^{4}}{\partial x^{4}}+\frac{\partial^{4}}{\partial y^{4}}\right), \quad R=\frac{2}{15}\left(\frac{\partial^{6}}{\partial x^{6}}+\frac{\partial^{6}}{\partial y^{6}}\right),
$$

the coordinate axis being parallel to the grid lines.

Applying (1) to determine the sum $\Delta u_{1}$ of the values of $\Delta u$ at the four nodes labelled 1 , we find

$$
h^{2} \Delta u_{1}=4 u_{0}-4 u_{1}+u_{2}+2 u_{3}+h^{4} Q u_{1}+O\left(h^{6}\right) .
$$

A finite difference expression for $h^{4} \Delta^{2} u_{0}$ may now be obtained by evaluating the Laplacian of $h^{4} \Delta u$ at the node 0 in accordance with (1) and using (4). Thus,

$$
\begin{aligned}
h^{4} \Delta^{2} u_{0} & =-4 h^{2} \Delta u_{0}+h^{2} \Delta u_{1}+h^{6} \Delta Q u_{0}+O\left(h^{8}\right) \\
& =20 u_{0}-8 u_{1}+u_{2}+2 u_{3}+h^{4} Q\left[-4 u_{0}+u_{1}\right]+h^{6} \Delta Q u_{0}+O\left(h^{8}\right) .
\end{aligned}
$$

By (1), the bracket in (5) equals $h^{2} \Delta u_{0}$ to within terms of the order $h^{4}$. Since $\Delta Q=Q \Delta$, we find

$$
h^{4} \Delta^{2} u_{0}=20 u_{0}-8 u_{1}+u_{2}+2 u_{3}+2 h^{6} Q \Delta u_{0}+O\left(h^{8}\right) .
$$

Analogous formulas in three dimensions may be obtained by the same technique. When the nodes are labelled as shown in Figs. 1 and 2, the simplest finite difference approximations to $\Delta u_{0}$ are

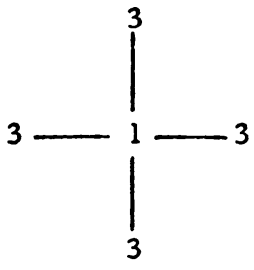

(a)

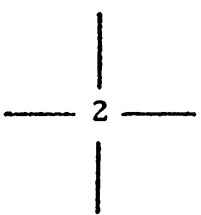

(b)

Fig. 2. Nodes of cubic grid: (a) Nodes in planes $z= \pm h$. (b) Nodes in plane $z= \pm 2 h$. For nodes in plane $z=0$, see Fig. 1 . 


$$
h^{2} \Delta u_{0}=-6 u_{0}+u_{1}+h^{4} S u_{0}+O\left(h^{6}\right)
$$

and

$$
12 h^{2} \Delta u_{0}=-90 u_{0}+16 u_{1}-u_{2}+h^{6} T u_{0}+O\left(h^{8}\right),
$$

where

$$
S=-\frac{1}{12}\left(\frac{\partial^{4}}{\partial x^{4}}+\frac{\partial^{4}}{\partial y^{4}}+\frac{\partial^{4}}{\partial z^{4}}\right), \quad T=\frac{2}{15}\left(\frac{\partial^{6}}{\partial x^{6}}+\frac{\partial^{6}}{\partial y^{6}}+\frac{\partial^{6}}{\partial z^{6}}\right)
$$

Using (7) in the manner in which (1) was used to derive (6), one readily obtains

$$
h^{4} \Delta^{2} u_{0}=42 u_{0}-12 u_{1}+u_{2}+2 u_{3}+2 h^{6} S \Delta u_{0}+O\left(h^{8}\right) .
$$

3. Hermitian approximations to $\Delta u=f$. Whereas formula (2) for $\Delta u_{0}$ has a higher degree of precision than formula (1), it involves the values of $u$ at the points 2 that have the distance $2 h$ from the point 0 . This means that even for a rectangular domain formula (2) cannot be used at interior points with the distance $h$ from the boundary. To avoid the necessity of constructing special formulas for these points near the boundary without sacrifice in the degree of precision, we may eliminate $u_{2}$ by combining the finite difference approximation to $12 \Delta u=12 f$ obtained from (2) with the one to $\Delta^{2} u=\Delta f$ obtained from (6). Replacing $\Delta u_{0}$ in (2) and (6) by $f_{0}$ and adding the resulting equations, we find

$$
h^{4} \Delta f_{0}+12 h^{2} f_{0}=-40 u_{0}+8 u_{1}+2 u_{3}+h^{6}(2 Q \Delta+R) u_{0}+O\left(h^{8}\right) .
$$

We now use the approximation (1) for $\Delta f_{0}$ and note that $Q f_{0}=Q \Delta u_{0}$. Thus,

$$
8 h^{2} f_{0}+h^{2} f_{1}=-40 u_{0}+8 u_{1}+2 u_{3}+h^{6}(Q \Delta+R) u_{0}+O\left(h^{8}\right) .
$$

The same technique may be applied in three dimensions. Eliminating $u_{2}$ by adding the finite difference approximations to $12 \Delta u=12 f$ and $\Delta^{2} u=\Delta f$ obtained from (8) and (10), we find

$$
h^{4} \Delta f_{0}+12 h^{2} f_{0}=-48 u_{0}+4 u_{1}+2 u_{3}+h^{6}(2 S \Delta+T) u_{0}+O\left(h^{8}\right) .
$$

Finally, evaluating $\Delta f_{0}$ by (7) and observing that $S f_{0}=S \Delta u_{0}$, we obtain

$$
6 h^{2} f_{0}+h^{2} f_{1}=-48 u_{0}+4 u_{1}+2 u_{3}+h^{6}(S \Delta+T) u_{0}+O\left(h^{8}\right) .
$$

Formulas (12) and (14) are well known; the first is due to Collatz [1] and the second to Albrecht [3]. The customary derivation of these formulas, however, is less direct than the one presented above.

Hermitian approximations of higher degrees of precision can be constructed in a similar manner.

\section{REFERENCES}

1. L. Collatz, Numerische Behandlung von Differentialgleichungen, Springer, Berlin, 1951; see also Das Mehrstellenverfahren bei Plattenaufgaben, Z. Angew. Math. Mech. 30, 385-388, and Einige neuere Forschungen über numerische Behandlung von Differentialgleichungen, Z. Angew. Math. Mech. 31, 230-236 (1951)

2. B. Meister and W. Prager, On the construction of symmetric difference operators for square and cubic lattices, Z. Angew. Math. Phys. 16, 403-410 (1965)

3. J. Albrecht, Taylor-Entwicklungen und finite Ausdrücke für $\Delta u$ und $\Delta \Delta u$. Z. Angew. Math. Mech. 33, 41-48 (1953) 